\title{
ABCG2 Polymorphism rs2231142 and hypothyroidism in metastatic renal cell carcinoma patients treated with sunitinib
}

Emilie Werbrouck, Julie Bastin, Diether Lambrechts, Annelies Verbiest, Thomas Van Brussel, Evelyne Lerut, Jean-Pascal Machiels, Vincent Verschaeve, Vincent Richard, Philip R. Debruyne, Brigitte Decallonne, Patrick Schöffski, Oliver Bechter, Pascal Wolter \& Benoit Beuselinck

To cite this article: Emilie Werbrouck, Julie Bastin, Diether Lambrechts, Annelies Verbiest, Thomas Van Brussel, Evelyne Lerut, Jean-Pascal Machiels, Vincent Verschaeve, Vincent Richard, Philip R. Debruyne, Brigitte Decallonne, Patrick Schöffski, Oliver Bechter, Pascal Wolter \& Benoit Beuselinck (2018): ABCG2 Polymorphism rs2231142 and hypothyroidism in metastatic renal cell carcinoma patients treated with sunitinib, Acta Clinica Belgica, DOI: 10.1080/17843286.2018.1477229

To link to this article: https://doi.org/10.1080/17843286.2018.1477229

Published online: 23 May 2018.

Submit your article to this journal ¿

a

View related articles $\asymp$

View Crossmark data $₫$ 


\title{
ABCG2 Polymorphism rs2231142 and hypothyroidism in metastatic renal cell carcinoma patients treated with sunitinib
}

\author{
Emilie Werbrouck ${ }^{a}$ (D), Julie Bastin ${ }^{a}$ (D) , Diether Lambrechts ${ }^{b, c}$, Annelies Verbiest ${ }^{\mathrm{a}}$, Thomas Van Brussel ${ }^{\mathrm{b}, \mathrm{c}}$, \\ Evelyne Lerut ${ }^{d}$, Jean-Pascal Machiels ${ }^{e}$, Vincent Verschaeve ${ }^{f}$ (D) Vincent Richard ${ }^{g}$, Philip R. Debruyne ${ }^{\text {hi, }}$, \\ Brigitte Decallonnej, Patrick Schöffski ${ }^{\mathrm{a}}$, Oliver Bechter ${ }^{\mathrm{a}}$, Pascal Wolter ${ }^{\mathrm{k}}$ and Benoit Beuselinck ${ }^{\mathrm{a}}$
}

\begin{abstract}
aDepartment of General Medical Oncology, University Hospitals Leuven, Leuven Cancer Institute and Department of Oncology, KU Leuven, Leuven, Belgium; 'Laboratory for Translational Genetics, Department of Oncology, KU Leuven, Leuven, Belgium; 'Research Center, VIB,

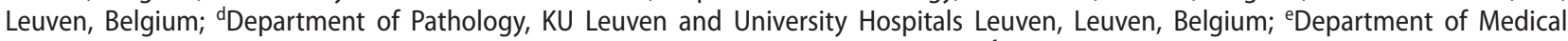
Oncology and Hematology, UCL Brussels and Hospitals Saint-Luc, Brussels, Belgium; 'Department of Medical Oncology, CHU Charleroi,

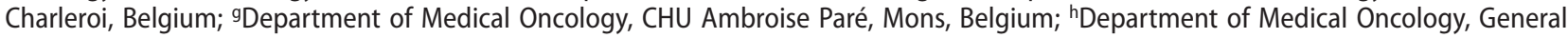
Hospital Groeninge, Kortrijk, Belgium; 'Faculty of Health, Social Care \& Education, Anglia Ruskin University, Chelmsford, UK; 'Department of Endocrinology, University Hospitals Leuven, Leuven, Belgium; 'Department of Medical Oncology, Centre Hospitalier Regional Verviers East Belgium, Verviers, Belgium
\end{abstract}

\begin{abstract}
Background and aim: Vascular endothelial growth factor receptor tyrosine kinase inhibitors (VEGFR-TKIs) cause significant adverse events including thyroid dysfunction, mainly hypothyroidism, in a considerable proportion of patients. In a series of metastatic renal cell carcinoma (mRCC) patients treated with sunitinib, we aimed to study the correlation between hypothyroidism and single nucleotide polymorphisms (SNPs) in genes involved in sunitinib pharmacokinetics and pharmacodynamics.

Patients and methods: We included 79 mRCC patients who started sunitinib between November 2005 and March 2016. Serum thyroid function markers were collected at start and during sunitinib therapy. Germ-line DNA genotyping for 16 SNPs in 8 candidate genes was performed. Endpoints were time to increase in thyroid stimulating hormone (TSH) and time to decrease in T4 or free T4 (FT4) on day 1 and day 28 of each sunitinib cycle.

Results: Patients with the ABCG2 rs2231142 CC-genotype had a significantly longer time-toTSH-increase on day 1 (11 vs. 5 cycles; $p=0.0011)$, and time-to-T4/FT4-decrease on day 1 (not reached vs. 10 cycles; $p=0.013$ ) and day 28 ( 28 vs. 7 cycles; $p=0.03$ ) compared to CA-carriers. Patients with the CYP3A5 rs776746 GG-genotype had a significantly longer time-to-TSH-increase at day 1 compared to GA-patients: 11 vs. 5 cycles $(p=0.0071)$. Significant associations were also found between PDGFRA rs35597368 and rs1800812 and time-to-TSH-increase at day 28.

Conclusion: Polymorphism rs2231142 in the efflux pump ABCG2 is associated with hypothyroidism in $\mathrm{mRCC}$ patients treated with sunitinib.
\end{abstract}

\section{KEYWORDS}

renal cell carcinoma; sunitinib; thyroid dysfunction; polymorphisms; efflux pumps

\section{Background}

Sunitinib is an oral multi-target inhibitor of vascular endothelial growth factor receptor 1, 2, and 3 (VEGFR 1, 2 and 3), FMS-like tyrosine kinase 3 (FLT3), colony stimulating factor 1 receptor (CSF1R), RET, KIT, and platelet-derived growth factor receptor (PDGFR). In 2007, a phase III trial demonstrated superior progression free survival (PFS) for sunitinib in comparison to interferon-alpha as first-line therapy in patients with clear cell mRCC (11 vs. 5 months). Based on these results, regulatory bodies in multiple countries granted approval for sunitinib in this indication $[1,2]$.

Thyroid dysfunction is a known adverse event of VEGFR-TKIs. In the majority of cases, hypothyroidism is observed, but also thyrotoxicosis can occur $[3,4]$. In the phase III COMPARZ trial, comparing first-line sunitinib with pazopanib, clinical hypothyroidism was observed in 135 out of 548 patients treated with sunitinib (24\%) [5]. Hypothyroidism has also been shown for other TKIs used to treat clear cell mRCC such as pazopanib (12\% of patients in the COMPARZ trial) [5], axitinib (19\% of patients in the axitinib pivotal phase III trial) [6], sorafenib (8\%) [6] and cabozantinib (20\%) [7]. However, an increase in TSH can be observed without T4/FT4-decrease, thus without the development of clinical hypothyroidism. This condition is called subclinical hypothyroidism. In smaller studies that focused on thyroid dysfunction during VEGFR-TKI treatment, higher incidences of TSH-increase were found. In a retrospective series of 66 RCC patients treated with sunitinib, 46 patients $(70 \%)$ showed elevated TSH values associated with decreased T4-levels in only 15 patients (23\%) [8]. 
In a prospective series of 59 patients with either mRCC or gastrointestinal stromal tumors treated with sunitinib, a similar proportion of patients (66\%) displayed thyroid dysfunction, defined as TSH-increase and/or T4 or T3-decrease [3].

The mechanism of VEGFR-TKI-induced thyroid dysfunction is still poorly understood [9]. Some authors suggest that sunitinib induces thyroiditis which could be immune-mediated (positive anti-thyroid peroxidase antibodies). Another hypothesis is that sunitinib, through the inhibition of VEGFR on thyroid follicular cells, causes capillary regression and thereby leads to thyroid dysfunction via this mechanism. A third possible explanation is a reduction in iodine uptake, needed for thyroid hormone synthesis, during treatment with sunitinib [10].

In a series of mRCC patients treated with sunitinib, we aimed to study the correlation between hypothyroidism and SNPs in genes involved in sunitinib pharmacokinetics and pharmacodynamics. If a SNP in a gene associated with pharmacokinetics has an impact on thyroid dysfunction, this gene has probably an impact on sunitinib plasma levels. If a SNP in a gene associated with sunitinib pharmacodynamics has an impact on thyroid dysfunction, this gene is possibly involved in the underlying mechanism of this adverse event.

\section{Materials and methods}

\section{Patient selection}

We enrolled mRCC patients who started sunitinib as first-line targeted therapy between November 2005 and March 2016 in participating hospitals. Only patients with available germ-line DNA and available data on thyroid function during therapy were included. Patients under thyroid hormone substitution at start of anti-VEGFRTKIs and individuals with baseline thyroid dysfunction were excluded. Sunitinib was given orally for four consecutive weeks followed by 2 weeks off per treatment cycle; treatment modifications followed local standards. Thus, at day 1, patients start the intake of sunitinib and at day 28, they stop the intake of sunitinib and start a therapeutic pause of 14 days. Patients were treated until disease progression or intolerable toxicity. Participating Belgian centers were the University Hospitals Leuven, Saint-Luc University Hospital (Brussels), the General Hospital Groeninge Kortrijk, Centre Hospitalier Universitaire (Charleroi), and Centre Hospitalier Universitaire Ambroise Paré (Mons). The study was approved by the local Ethical Committee of each participating hospital.

\section{Endpoints}

In the participating centers, thyroid function (TSH, T4, and/or FT4) was routinely tested on day 1 and day 28 of each sunitinib treatment cycle. We collected data on thyroid function by reviewing electronic medical records. Laboratory reference ranges were $0.27-$ $4.2 \mathrm{mIU} / \mathrm{l}$ for $\mathrm{TSH}, 5.1-14.1 \mu \mathrm{g} / \mathrm{dl}$ for total $\mathrm{T} 4$, and 12-22 pmol/l for free T4 (FT4). In most of the patients, T4 was measured; in a minority of the patients, FT4 was measured according to local hospital standards. Patients who started with thyroid hormone substitution because of rising TSH-values were censored at that moment for TSH and T4/FT4-levels. Patients who stopped sunitinib were also censored at that moment.

Our primary endpoints were time-to-TSH-increase on day 1 and day 28 and time-to-T4/FT4-decrease day 1 and 28. Time-to-TSH-increase was defined as the time from the start of sunitinib till the first increase of TSH above the upper limit of normal. Time-to-T4/FT4decrease was defined as the time from start of sunitinib till the first decrease of T4/FT4 under the lower limit of normal. Secondary endpoints were the incidence of subclinical (also called mild) and clinical (or overt) hypothyroidism. Subclinical hypothyroidism was defined as increased TSH with (F)T4-levels in the normal range. Clinical hypothyroidism was defined as increased TSH with decreased (F)T4-levels. We did not take clinical symptoms into account. We preferred a time-to-event approach because it allows us to calculate for each patient the precise time to TSH-increase and to T4/FT4decrease. As such, it is more precise than an estimation of thyroid dysfunction at a predefined single moment, for instance after three cycles.

\section{SNP selection}

Candidate SNPs $(n=16)$ were selected based on the scientific literature. Table 1 reports all the SNPs that were studied.

We selected SNPs in three genes involved in sunitinib pharmacokinetics: the efflux pumps ATP binding cassette member B1 (ABCB1) (rs1045642, rs1128503, rs2032582) and G2 (ABCG2) (rs2231142) and in cytochrome CYP3A5 (rs776746) [11]. Sunitinib is recognized, bound, and can be effluxed, for instance from enterocytes to the bowel lumen, in a specific concentration window by ABCB1 and ABCG2 [12-14]. Efflux pumps are present on epithelial cells of the small intestine and are part of our 'chemo-immunity' system. Their function is to efflux substances that are entering through osmosis in the enterocyte and then into the blood circulation, back to the lumen of the bowel. These efflux pumps are also present in the biliary tract and on the blood-brain barrier. Data on correlations with sunitinib plasma levels, dose reductions, adverse events, and outcome for these different SNPs [15-23] are available in literature.

We selected SNPs in five genes involved in sunitinib pharmacodynamics. SNPs in PDGFRA (rs1800812, rs35597368), VEGFR1 (rs9582036, rs9554320), and VEGFR3 (rs307821, rs307826) were selected because the 
Table 1. Genotype and allele distribution of the genotyped SNPs.

\begin{tabular}{|c|c|c|c|c|c|c|c|c|c|}
\hline Gene & RS ID & Polymorphism & $\begin{array}{l}\text { Location or } \\
\text { functional } \\
\text { consequence }\end{array}$ & $\mathrm{N}$ & $\begin{array}{l}\text { Wildtype/ } \\
\text { Wildtype } \\
\text { n (\%) }\end{array}$ & $\begin{array}{c}\text { Wildtype/ } \\
\text { Variant n (\%) }\end{array}$ & $\begin{array}{l}\text { Variant/ } \\
\text { Variant n } \\
(\%)\end{array}$ & $\begin{array}{c}\text { Observed } \\
\text { minor allele } \\
\text { frequency (\%) }\end{array}$ & $\begin{array}{l}\text { Minor allele } \\
\text { frequency in } \\
\text { dbSNP (\%) }\end{array}$ \\
\hline \multicolumn{10}{|c|}{ Sunitinib pharmacokinetics } \\
\hline \multirow[t]{3}{*}{ ABCB1 } & rs1045642 & $3435 C>T$ & $|1154|$ & 79 & 26 & 33 & 20 & 46.2 & $51 \%(\mathrm{~T})$ \\
\hline & rs1128503 & $1236 C>T$ & G412G & 79 & 24 & 39 & 16 & 44.9 & $41.6 \%(\mathrm{~T})$ \\
\hline & rs2032582 & $2677 \mathrm{G}>\mathrm{T}$ or $\mathrm{G}>\mathrm{A}$ & A893S & 79 & 24 & 27 & 18 & 39.9 & $43.5 \%(\mathrm{~T})$ \\
\hline CYP3A5 & rs776746 & $6986 G>A$ & $\begin{array}{l}\text { Affecting } \\
\text { splicing }\end{array}$ & 79 & 66 & 13 & 0 & 8.2 & $5.7 \%(\mathrm{~A})$ \\
\hline $\mathrm{ABCG} 2$ & rs2231142 & $421 C>A$ & Q141K & 79 & 58 & 21 & 0 & 13.3 & $9.1 \%(A)$ \\
\hline \multicolumn{10}{|c|}{ Sunitinib pharmacodynamics } \\
\hline \multirow[t]{2}{*}{ PDGFRA } & rs35597368 & $1580 \mathrm{~T}>\mathrm{C}$ & S478P & 78 & 64 & 14 & 0 & 8.9 & $10.7 \%(C)$ \\
\hline & rs1800812 & $-537 \mathrm{G}>\mathrm{T}$ & promotor & 75 & 58 & 16 & 1 & 12 & $20.8 \%(\mathrm{~T})$ \\
\hline \multirow[t]{2}{*}{ VEGFA } & rs699947 & $-2578 \mathrm{C}>\mathrm{A}$ & Promotor & 78 & 18 & 39 & 21 & 48.1 & $51.9 \%(A)$ \\
\hline & rs2010963 & $+405 G>C$ & & 73 & 35 & 30 & 8 & 31.5 & $30.8 \%(C)$ \\
\hline \multirow[t]{2}{*}{ VEGFR1 } & rs9582036 & $-2578 C>A$ & & 79 & 49 & 24 & 6 & 22.8 & $27.2 \%(C)$ \\
\hline & rs 9554320 & $692 A>C$ & intron & 79 & 32 & 32 & 15 & 39.2 & $54.4 \%(C)$ \\
\hline \multirow[t]{2}{*}{ VEGFR3 } & rs307821 & $3971 \mathrm{G}>\mathrm{T}$ & R1324L & 79 & 56 & 21 & 2 & 15.8 & $8.9 \%(\mathrm{~T})$ \\
\hline & rs307826 & $1480 A>G$ & T494A & 79 & 59 & 18 & 2 & 13.9 & $10.7 \%(\mathrm{G})$ \\
\hline \multicolumn{10}{|c|}{ Thyroid function } \\
\hline \multirow[t]{3}{*}{ RET } & rs1799939 & $2071 G>A$ & G619S & 78 & 47 & 26 & 5 & 23.1 & $18.9 \%(\mathrm{~A})$ \\
\hline & rs 1800861 & $2307 T>G$ & L769L & 77 & 51 & 21 & 5 & 20.1 & $23.6 \%(\mathrm{G})$ \\
\hline & rs1800863 & $2712 C>G$ & S904S & 77 & 47 & 25 & 5 & 22.7 & $19.2 \%(\mathrm{G})$ \\
\hline
\end{tabular}

Notes: For most of the SNPs, the minor allele frequency as reported on dbsnp (https://www.ncbi.nlm.nih.gov/SNP) for large patients series in Europe was similar to the minor allele frequency found in our patient series. However, for one SNPs (rs9554320), the difference of minor allele frequency was above $10 \%$. This difference is probably due to the small series in our study. The SNPs were successfully genotyped with success rates $\geq 92.4 \%$ for each SNP and an overall average success rate of $99 \%$.

corresponding proteins are typical therapeutic targets of sunitinib and are involved in normal blood vessels physiology, including thyroid blood vessels. SNPs in these genes have been associated with tumor response on VEGFR-TKIs $[15,17,24]$. Two SNPs in VEGFA (rs699947, rs2010963) were selected because of previously reported associations between these SNPs and adverse events [17,25]. Three SNPs were selected in RET (rs1799939, rs1800861, rs1800863) because RET is a target of sunitinib and plays a role in thyroid physiology. These SNPs have been correlated with susceptibility to thyroid cancer [26].

\section{SNP genotyping}

DNA was isolated from fresh frozen normal kidney tissue sampled during nephrectomy using the Qiaquick extraction kit (Qiagen, Valencia, CA) and quantified by fluorometry (Fluoroskan Thermo Labsystems, CergyPontoise, France). DNA was isolated from peripheral blood with the Qiagen DNA kit (Qiagen, Valencia, CA) and final DNA concentration quantified with Nanodrop (Nanodrop, Wilmington, UDE). For the genotyping on germ-line DNA samples, high-throughput SNP genotyping was performed using the Sequenom MassArray platform (Sequenom, San Diego, CA) [27]. Genotyping analysis was performed by investigators blinded for the clinical data. The variants in each SNP were analyzed in the same combinations as in previous publications, according to dominant, recessive, or co-dominant genetic models. For SNPs in RET, the effect on thyroid dysfunction was studied for the three combinations and overlapping curves were pooled if possible.

\section{Statistical analysis}

Time to thyroid dysfunction for different genotypes was estimated with the Kaplan-Meier method. Curves were compared with log-rank test and $p$-values of $<0.05$ were considered as significant. Bonferroni correction was applied for multiple testing. As the impact of 16 SNPs was analyzed, we considered a $p$-value of 0.003 (0.05 divided by 16) as threshold for significance after correction for multiple testing. Baseline TSH and T4/ FT4-levels were compared between subgroups of patients with distinct genotypes with a student t-test. Fisher Exact test was used for comparison of percentages. Moreover, we searched in the literature for parallel findings and for possible underlying mechanisms to strengthen our findings.

\section{Results}

\section{Patient characteristics}

We included 79 mRCC patients who started sunitinib between November 2005 and March 2016. Fourteen patients could not be included in the study because of preexisting thyroid dysfunction. Among them, 6 patients were treated with thyroid supplementation at start of sunitinib, 4 had an increased TSH, and 3 a low TSH at the start of the first sunitinib cycle. One patient was treated with the anti-thyroid drug thiamazol. The characteristics of the included patients are shown in Table 2. Among the remaining 79 included patients, 56 were men and 23 women with a median age of 63 years (range 27-84). Tumor histology was clear cell RCC in 71 patients, papillary type in 7 patients, and chromophobe 
Table 2. Included patients.

\begin{tabular}{|c|c|c|}
\hline Included patients $(n=79)$ & & \\
\hline At initial diagnosis & & \\
\hline $\begin{array}{l}\text { Median age (years) (range) } \\
\text { Male } \\
\text { M1 at diagnosis } \\
\text { Fuhrman Grade } 4 \\
\text { Sarcomatoid dedifferentiation } \geq 25 \%\end{array}$ & & $\begin{array}{c}63 \text { (range } 27-84) \\
71 \%(56 / 79) \\
49 \%(39 / 79) \\
51 \%(38 / 75) \\
3 \%(2 / 68)\end{array}$ \\
\hline At start of sunitinib & & \\
\hline $\begin{array}{l}\text { Karnofsky Performance Status } \leq 70 \\
\text { Neutrophils }>7.800 / \mathrm{mm}^{3} \\
\text { Platelets }>450.000 / \mathrm{mm}^{3} \\
\text { Hemoglobin }<12.0 \mathrm{~g} / \mathrm{dl} \text { (women) or }<14.0 \mathrm{~g} / \mathrm{dl} \text { (men) } \\
\text { Lactate dehydrogenase }>1.5 \mathrm{x} \text { ULN } \\
\text { Corrected calcium }>10 \mathrm{mg} / \mathrm{dl} \\
\text { Time nephrectomy to systemic treatment }<12 \text { months } \\
\text { Immunotherapy before sunitinib }\end{array}$ & & $\begin{array}{l}14 \%(11 / 78) \\
5 \%(4 / 79) \\
13 \%(10 / 79) \\
51 \%(40 / 79) \\
1 \%(1 / 79) \\
13 \%(10 / 77) \\
58 \%(46 / 79) \\
10 \%(8 / 79)\end{array}$ \\
\hline Site of metastasis & $\begin{array}{l}\text { Lung } \\
\text { Liver } \\
\text { Bone } \\
\text { Brain }\end{array}$ & $\begin{array}{c}63 \%(50 / 79) \\
23 \%(18 / 79) \\
44 \%(35 / 79) \\
6 \%(5 / 79)\end{array}$ \\
\hline Median number of metastatic sites & & 2 \\
\hline IMDC prognostic score & $\begin{array}{l}\text { Favorable } \\
\text { Intermediate } \\
\text { Poor }\end{array}$ & $\begin{array}{l}24 \%(19 / 78) \\
53 \%(41 / 78) \\
23 \%(18 / 78)\end{array}$ \\
\hline Histological subtype & $\begin{array}{l}\text { Clear cell } \\
\text { Papillary } \\
\text { Chromophobe }\end{array}$ & $\begin{array}{c}90 \%(71 / 79) \\
9 \%(7 / 79) \\
1 \%(1 / 79)\end{array}$ \\
\hline
\end{tabular}

Notes: IMDC = international metastatic renal cell carcinoma database consortium; ULN = upper limit of normal.

RCC in 1 patient. Eight patients had previously received interferon-based immunotherapy.

All 79 patients received at least one cycle of sunitinib, and 78 patients received more than 1 cycle of sunitinib. The median follow-up was 61 months, ranging from 2 to 129 months. The median overall survival (OS) was 29 months, while the median progression free survival (PFS) was 19 months.

Main reasons for drug discontinuation were disease progression $(n=51,65 \%)$ and treatment-related toxicity $(n=13,16 \%)$. In one patient, sunitinib was stopped because of general deterioration and in one patient because of complete remission. One patient deceased. Sunitinib dose reductions from 50 to $37.5 \mathrm{mg} / \mathrm{d}$ occurred in 47 out of 79 patients on a four weeks on, two weeks off schedule. Median time-to-dose reduction from 50 to $37.5 \mathrm{mg} / \mathrm{d}$ was 5 cycles. Main toxicities leading to dose reductions were diarrhea and hand-foot syndrome. Less frequent were asthenia and thrombocytopenia. A consecutive sunitinib dose reduction from 37.5 to $25 \mathrm{mg} / \mathrm{d}$ occurred in 17 patients.

\section{Evolution of thyroid function during sunitinib therapy}

Only 13 patients (16\%) remained euthyroid during sunitinib therapy. Twenty-six patients (33\%) developed clinical/overt and 36 (46\%) subclinical/mild hypothyroidism. Five patients $(6 \%)$ developed thyrotoxicosis with decreased TSH-levels and increased T4/FT4-levels.

The evolution of TSH and T4/FT4-levels after start of sunitinib in the total patient series is reported in Figure 1, panel A. As expected, TSH-levels rose well before any decrease in peripheral thyroid hormone levels occurred. The first event to occur is TSH-increase on day 28 , after a median of only two cycles (range 1-21). The next event is TSH-increase on day 1 , which occurred after a median of nine cycles (range 2-23). The final event is a decrease in T4/FT4-levels on day 28 and day 1, after a median treatment duration of 22 cycles (range 1-28 and range $5-22$, respectively). The fact that $\mathrm{TSH}$-increase on day 1 occurs later than TSH-increase on day 28 shows that there is a recovery of thyroid function during the 2 weeks off-treatment. On the contrary, T4/FT4-decrease seems not to recover during the 2 weeks off-treatment. Twenty-six out of the 29 patients (90\%) who had low T4/FT4 levels at a given moment had previously elevated TSH at day 28 but only $8(28 \%)$ had a previously elevated TSH on day 1. It is important to notice that the first sunitinib dose reduction (from $50 \mathrm{mg}$ to $37.5 \mathrm{mg}$ ) occurred after a median treatment period of 5 cycles (Figure 1, panel B). As a consequence, TSH-increase at day 28 , occurring after a median treatment period of 2 cycles, occurred earlier that the first dose reduction. This lowers the impact that dose reductions can have on TSH-increase at day 28.

\section{Correlations between SNPs and thyroid dysfunction (Table 3)}

\section{Impact of rs 2231142 in ABCG2}

As there were no patients with the AA-genotype in our series, we compared patients with the AC- and the CC-genotype. Compared to AC-carriers, CC-carriers had a significantly longer time-to-TSH-increase on day 1 (11 vs. 5 cycles; $p=0.0013$ ), and time-to-T4/FT4-decrease 


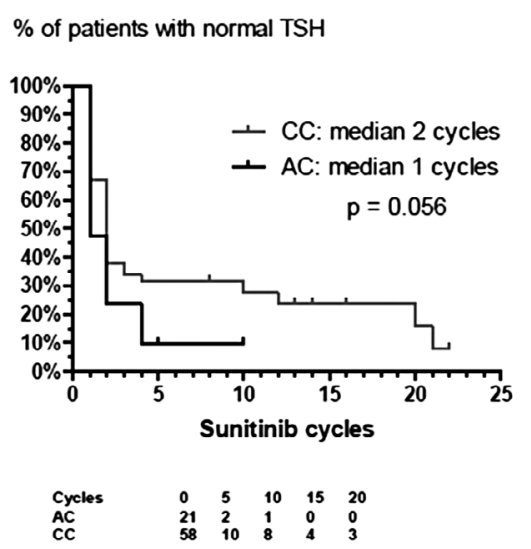

Time-to-T4/FT4-decrease on day 1 (ABCG2 rs2231142)

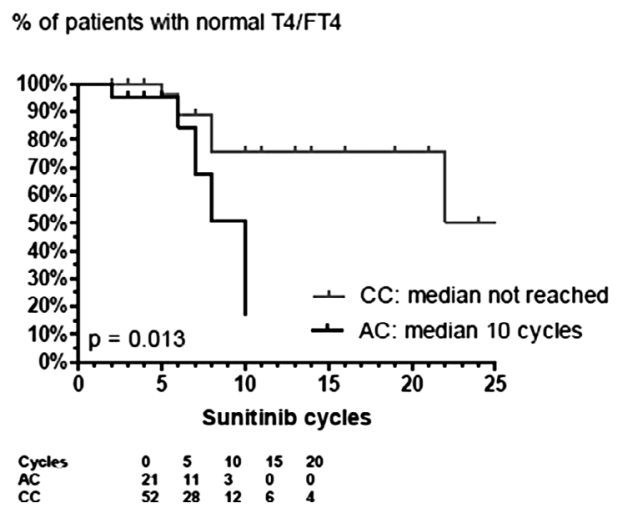

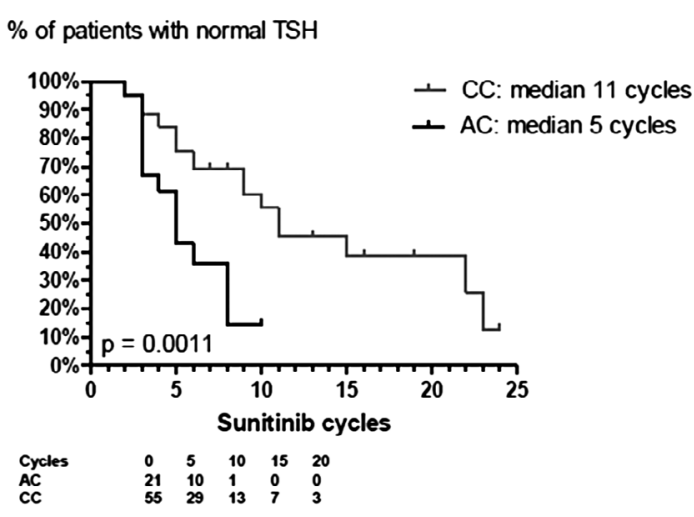

Time-to-T4/FT4-decrease on day 28 (ABGC2 rs2231142)

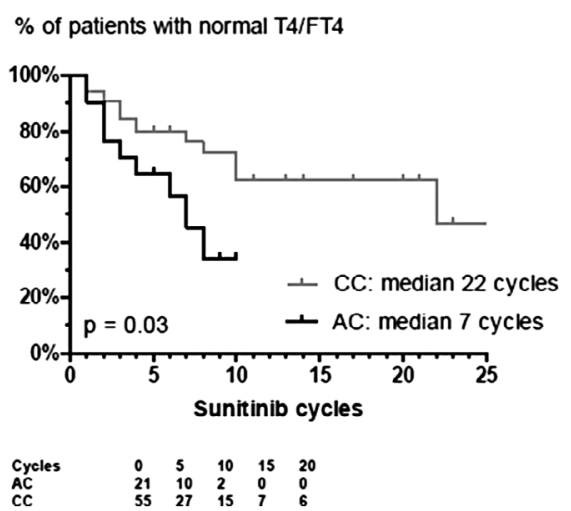

Figure 1. Panel A: Cumulative thyroid dysfunction (TSH-increase on day 28 and day 1, T4/FT4-decrease on day 28 and day 1) during sunitinib therapy. Panel B: comparison between time-to-TSH-increase on day 28 and time-to-first-dose-reduction.

Table 3. Univariate analysis: association between SNPs and thyroid dysfunction.

\begin{tabular}{|c|c|c|c|c|c|c|c|c|c|}
\hline \multirow[b]{2}{*}{ SNP } & \multirow[b]{2}{*}{ Geno-type } & \multicolumn{2}{|c|}{$\begin{array}{l}\text { Time to TSH increase day } \\
28 \text { (cycles) }\end{array}$} & \multicolumn{2}{|c|}{$\begin{array}{c}\text { Time to T4/FT4 decrease } \\
\text { day } 28\end{array}$} & \multicolumn{2}{|c|}{$\begin{array}{l}\text { Time to TSH increase day } \\
1 \text { (cycles) }\end{array}$} & \multicolumn{2}{|c|}{$\begin{array}{c}\text { Time to T4/FT4 decrease } \\
\text { day } 1\end{array}$} \\
\hline & & Median & $p$-value & Median & $p$-value & Median & $p$-value & Median & $p$-value \\
\hline Total series & & \multicolumn{2}{|c|}{2} & \multicolumn{2}{|c|}{22} & \multicolumn{2}{|c|}{9} & \multicolumn{2}{|c|}{22} \\
\hline ABCG2 & $A C$ & 1 & 0.056 & 7 & 0.03 & 5 & 0.0011 & 10 & 0.013 \\
\hline rs2231142 & $\mathrm{CC}$ & 2 & & 28 & & 11 & & NR & \\
\hline CYP3A5 & AG & 2 & 0.45 & NR & 0.85 & 5 & 0.0071 & NR & 0.9 \\
\hline rs776746 & GG & 2 & & 2 & & 11 & & 2 & \\
\hline PDGFRA & GG & 2 & 0.039 & 10 & 0.22 & 10 & 0.88 & 22 & 0.97 \\
\hline rs1800812 & GT-TT & 3 & & NR & & 9 & & NR & \\
\hline PDGFRA & $\mathrm{TT}$ & 2 & 0.01 & 22 & 0.5 & 9 & 0.97 & 22 & 0.8 \\
\hline \multirow[t]{2}{*}{ rs35597368 } & $\mathrm{TC}$ & 12 & & NR & & 9 & & NR & \\
\hline & $\mathrm{CC}$ & 2 & & NR & & 8 & & NR & \\
\hline
\end{tabular}

Notes: Significant associations are expressed in bold.

NR: not reached.

on day 1 (not reached vs. 10 cycles; $p=0.013$ ) and day 28 (28 vs. 7 cycles; $p=0.03$ ). Time-to-TSH-increase on day 28 showed a trend in the same direction $(p=0.056)$ (Figure 2). The association with time-to-TSH-increase on day 1 remained significant after Bonferroni correction for multiple testing. A higher prevalence of the AA/ AC-genotype was found in patients with hypothyroidism $(31 \%)$ compared to patients without hypothyroidism (14\%), but this difference was not statistically significant. There was no impact of rs2231142 on baseline TSH or $\mathrm{T} 4$ values. There was no significant difference in time to dose reduction $(50>37.5 \mathrm{md} /$ day and $37.5>25 \mathrm{mg}$ / day) between the genotypes.

\section{Impact of rs776746 in CYP3A5}

As the AA-genotype was not represented in this patient series, we analyzed AG- vs. GG-carriers. Compared to AG-carriers, GG-carriers had a significantly longer timeto-TSH-increase at day 1 (11 vs. 5 cycles; $p=0.0071$ ) (Figure 3). Nevertheless, there was no significant correlation with TSH-increase at day 28 or T4/FT4-decrease at day 1 or day 28. There was no impact of rs776746 

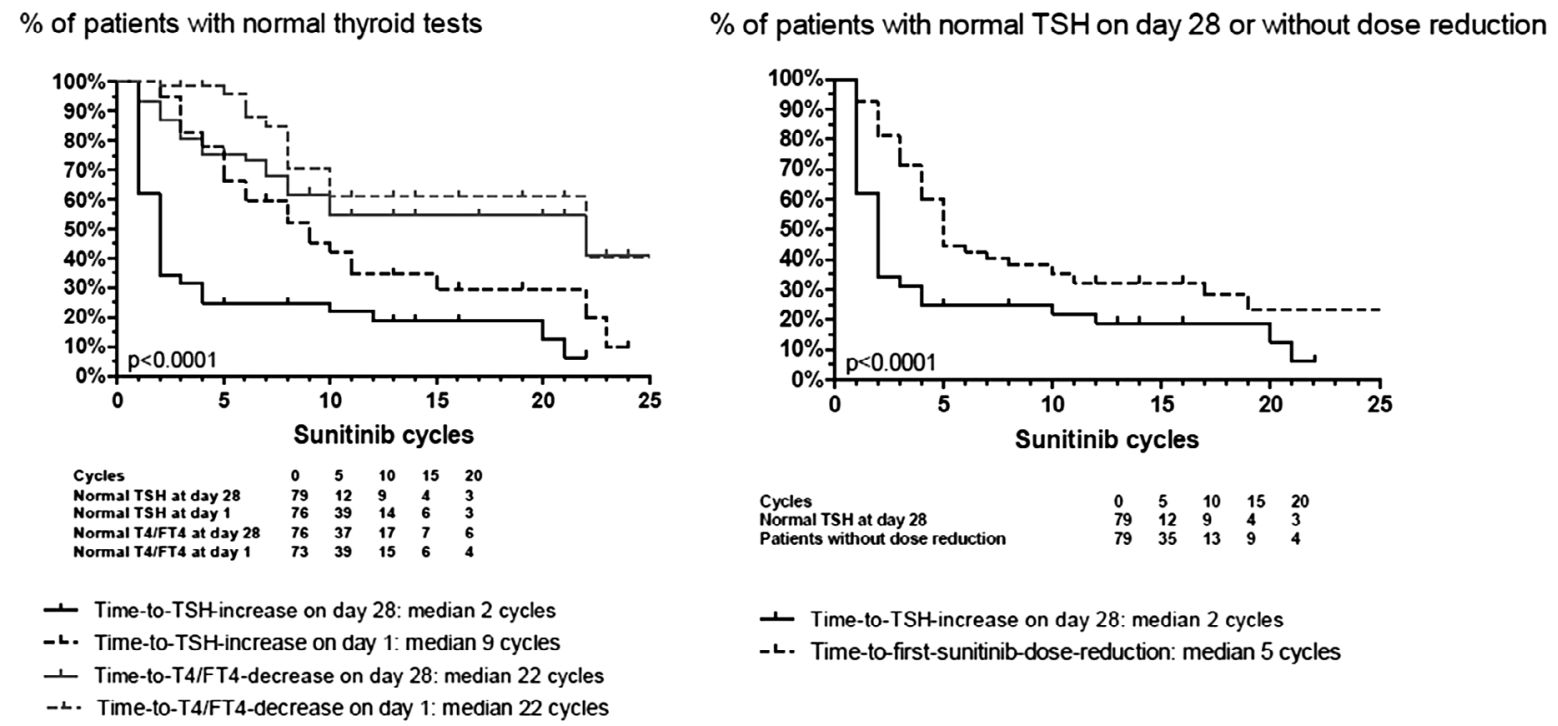

Figure 2. Cumulative thyroid dysfunction (TSH-increase on day 28 and day 1,T4/FT4-decrease on day 28 and day 1) during sunitinib therapy.

Time-to-TSH-elevation on day 1 (CYP3A5 rs776746)

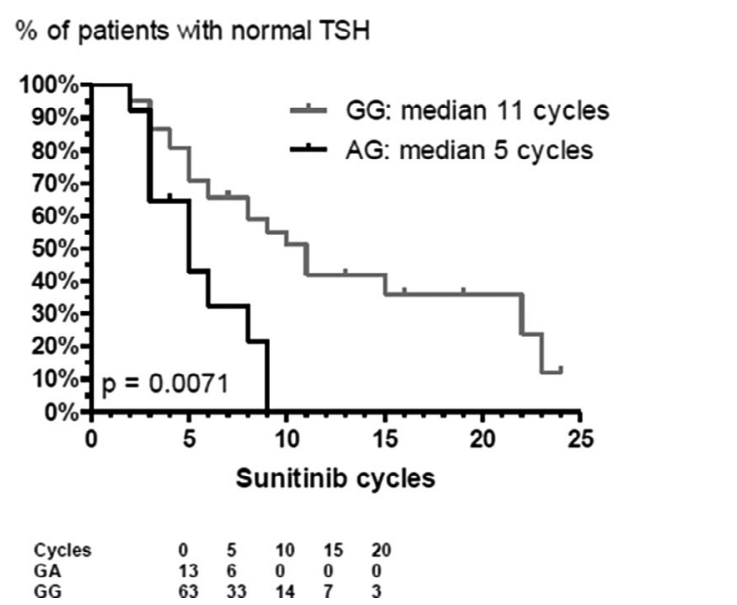

Figure 3. Association of rs776746 in CYP3A5 and TSH-increase on day 1.

on baseline TSH values. There was no significant difference in time to dose reduction $(50>37.5 \mathrm{md} /$ day and $37.5>25 \mathrm{mg} /$ day) between the genotypes.

\section{Impact of rs 35597368 and rs 1800812 in PDGFRA}

As the rs 1800812 TT-genotype was only present in one patient, this patient was pooled with the GT-carriers and the pooled data were compared with the data of the GG-carriers. For rs35597368, there were only TT- and TC-carriers. The rs35597368 TC-genotype was found to be associated with a longer time-to-TSH-increase at day 28 ( 12 cycles vs. 2 cycles; $p=0.0016$ ). Similarly, the rs 1800182 GT/TT-genotype was found to be associated with a longer time-to-TSH-increase at day 28 ( 3 cycles vs. 2 cycles; $p=0.039$ ) (Figure 4$)$. Both SNPs are in linkage disequilibrium: most patients who are rs1800182 GT/ TT-carriers were also rs35597368 TC-carriers. However, no impact on TSH-increase at day 1 nor on T4/FT4decrease could be observed. There was no impact of rs35597368 or rs 1800812 on baseline TSH values.

We could not find any other significant association between polymorphisms in ABCB1, VEGFA, VEGFR1, VEGFR3, or RET and thyroid dysfunction.

\section{Discussion}

We aimed to study the correlation between subclinical and clinical hypothyroidism and SNPs in genes involved in sunitinib pharmacokinetics and pharmacodynamics.

SNP rs2231142 in efflux pump ABCG2 was found to be correlated to the development of subclinical and clinical hypothyroidism. The CC-genotype has a protective effect, while patients with the AC-genotype developed earlier thyroid dysfunction. Minor findings are correlations between SNP rs776746 in CYP3A5 and time-to-TSH-increase at day 1 and SNPs rs35597368 and rs 1800812 in PDGFRA and time-to-TSH-increase at day 28.

\section{Correlation with SNP rs2231141 in ABCG2}

Patients with the ABCG2 rs2231142 CC-genotype had a significantly longer time-to-TSH-increase, and a longer time-to-T4/FT4-decrease. The impact on thyroid function starts already after a median of 2 cycles, while the first dose reduction occurred significantly later, after a median of 5 cycles.

There are several parallel findings in the literature showing associations between ABCG2 rs2231142 and sunitinib-induced toxicities in mRCC patients (Table 4). In a series of 333 patients, dose reductions and hypertension were more frequently observed in AA/AC-carriers compared to CC-carriers [22]. In a series of 219 patients, the A-allele was associated with thrombocytopenia and 

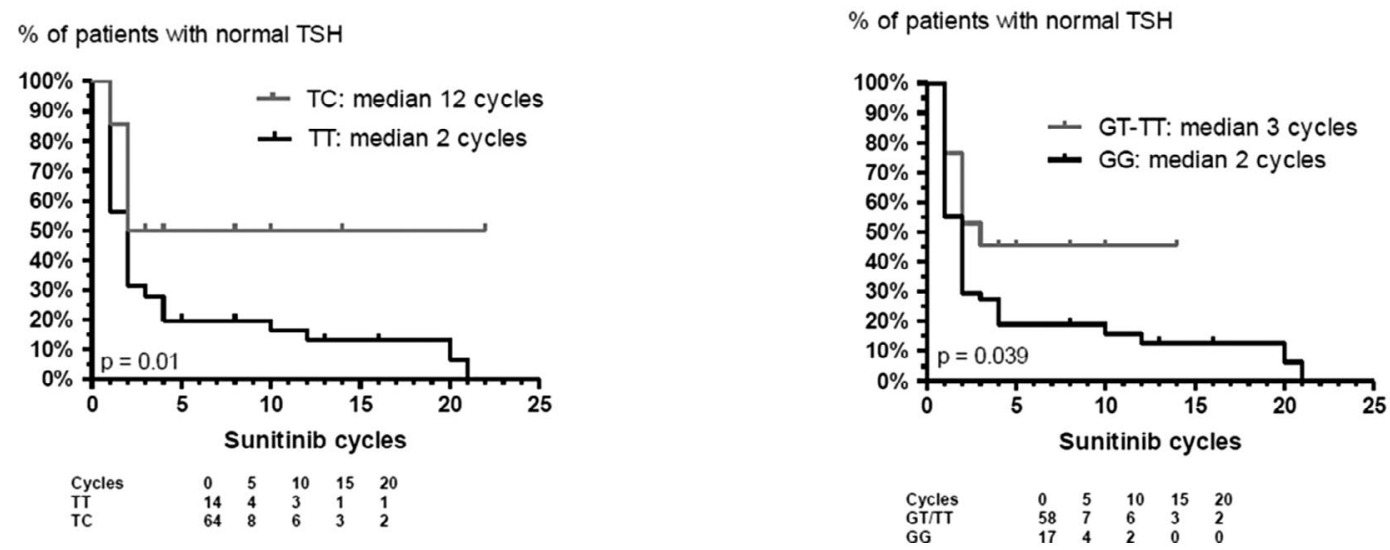

Figure 4. Association of rs35597368 and rs 1800812 in pdgfra and TSH-increase on day 28.

Table 4. Overview of the impact of rs2231142 on sunitinb plasma levels or on adverse events.

\begin{tabular}{|c|c|c|c|}
\hline \multicolumn{4}{|c|}{ Plasma levels/clearance } \\
\hline Narjoz et al. [19] & $N=92$ & AA: Higher plasma levels compared to AC/CC & $p=0.014$ \\
\hline Mizuno et al. [29] & $N=19$ & AA: Higher plasma levels compared to CC & $p=0.02$ \\
\hline Diekstra et al. [23] & $N=107$ & CC: Higher plasma clearance compared to AC & Not significant \\
\hline \multicolumn{4}{|c|}{ Dose reductions or sunitinib withdrawal } \\
\hline Diekstra et al. [22] & $N=333$ & A-allele: More dose reductions after cycle 1-2-3 & $p=0.022$ HR $0.36(0.15-0.86)$ \\
\hline \multicolumn{4}{|l|}{ Adverse events } \\
\hline Werbrouck et al. & $N=79$ & $\begin{array}{l}\text { AC: Earlier TSH-increase compared to CC } \\
\text { AC: Earlier T4-decrease compared to CC }\end{array}$ & \\
\hline Low et al. [20] & $N=219$ & $\begin{array}{l}\text { A-allele: More thrombocytopenia. } \\
\text { A-allele: More liver injury. }\end{array}$ & $\begin{array}{l}\text { HR } 1.86(95 \% \mathrm{Cl} 1.17-2.94) \\
\text { HR } 2.18(95 \% \mathrm{Cl} 1.03-4.64)\end{array}$ \\
\hline Kim et al. [21] & $N=65$ & $\begin{array}{l}\text { AA: More thrombocytopenia. } \\
\text { AA: More hand-foot-skin-reaction. }\end{array}$ & $\begin{array}{l}\text { HR } 9.90 \text { (1.16-infinity) } \\
\text { HR } 28.5 \text { (95\% Cl 2.22-364.9) }\end{array}$ \\
\hline Diekstra et al. [22] & $N=333$ & A-allele: More hypertension & HR 0.03 (95\% Cl 0.001-0.85) \\
\hline Chu et al. [28] & $N=86$ & $\mathrm{AA}$ : Less frequent neutropenia compared to $\mathrm{AC} / \mathrm{CC}$ & $p=0.03$ \\
\hline
\end{tabular}

liver injury. Moreover, a higher prevalence of the AA/ AC-genotype was found in patients with hypothyroidism (59\%) compared to patients without hypothyroidism (48\%), but this difference was not statistically significant [20]. Among 65 patients, grade 3 or 4 thrombocytopenia, neutropenia, and hand-foot syndrome were more frequently observed in AA-carriers [21]. On the contrary, Chu et al. showed in 86 patients that AA-carriers had a lower incidence (36\%) of neutropenia compared to AC- and CC-carriers (55\%) [28].

The underlying mechanism of increased toxicity probably increased sunitinib plasma levels (Table 4). In a series of 92 patients treated with sunitinib, higher plasma levels were found in AA-carriers. In these patients, higher plasma levels were associated with increased grade $\geq 3$ toxicity. Elevated SU12662 (the main sunitinib metabolite) plasma levels were associated with grade $\geq 2$ thrombocytopenia [19]. Similarly, Mizuno et al. have shown higher plasma levels in 10 AA-carriers compared to 9 CC-carriers. Thrombocytopenia and hypertension and poor compliance were associated with systemic exposure to sunitinib and its active metabolite [29]. Finally, Diekstra et al. have shown a trend toward a higher sunitinib clearance in CC-carriers compared to AC-carriers [23]. In summary, most of these findings are coherent and point toward higher plasma levels and more adverse events in AA/AC-carriers compared to CC-carriers.

rs2231142 $(421 \mathrm{C}>\mathrm{A})$ is a missense polymorphism leading to a Q141 K amino acid alteration. Possibly, the amino acid replacement in the ABCG2 results in functional impairment and may cause increased oral absorption of sunitinib followed by more severe toxic effects [30]. The A-allele and the AA-genotype are more frequent in the Asiatic population compared to the Caucasian population.

\section{Correlation with SNP rs776746 in CYP3A5}

Our findings on the impact of rs776746 in CYP3A5 on hypothyroidism (GG-carriers having a significantly longer time-to-TSH-increase at day 1 compared to GA-carriers) are also in coherence with other published observations in mRCC patients treated with sunitinib. In a series of 95 patients, more frequent dose reductions were found in GA-carriers compared to GG-carriers [17]. In a series of 333 patients, more frequent dose reductions in AA/AG-carriers compared to GG-carriers were shown [22]. In one publication, the AA/AG-genotype was associated with improved PFS (not reached in AA/AG-carriers vs. 9.3 months in GG-carriers) [31]. These findings are coherent and 
point toward higher plasma levels, better efficacy, and more adverse events in AA/AG-carriers compared to GG-carriers, through reduced sunitinib metabolism and higher plasma levels. CYP3A5 is a metabolizing liver enzyme, possibly involved in sunitinib metabolism. Surprisingly, Diekstra et al. have found a 19\% higher sunitinib clearance in AA/AG-carriers compared to GG-carriers. Nevertheless, the principle metabolite of sunitinib, SU12662, has a longer half-life compared to sunitinib. Thus, the increased availability of SU12662 could be responsible for increased toxicity and efficacy in AA/AG-carriers compared to GG-carriers [23].

\section{Correlation with SNPs rs35597368 and rs 1800182 in PDGFRA}

The PDGFRA rs35597368 TC- and rs1800182 GT/ TT-genotype were found to be associated with a longer time-to-TSH-increase at day 28. There are no data available in the literature on correlations between these SNPs and sunitinib plasma levels or induced toxicities and there is no suspected underlying mechanism. Further studies are warranted to exclude type I errors.

\section{Negative findings}

Surprisingly, we could not find any significant association between polymorphisms in ABCB1 and hypothyroidism. In recent years, several authors have reported correlations between rs1045642, rs1128503, and rs2032582 in ABCB1 and sunitinib exposure, clearance and dose reductions, as well as outcome $[15,22,31]$. Higher sunitinib exposure and more rash and mucositis were observed in patients with the rs 1045642 CC-genotype [32]. A 17\% higher sunitinib clearance in rs1128503 TT-carriers and a 18\% higher sunitinib clearance in rs2032582 TT-carriers were described [22]. Hypertension and hand-foot syndrome were correlated with the rs2032582 GG-genotype [33]. Dose reduction was delayed in rs1128503 TT-carriers [16]. However, our patient series was small and thyroid function, as well as other adverse events and plasma levels, can be influenced by several additional factors.

We could not find any significant association between polymorphisms in VEGFA, VEGFR1, VEGFR3, or RET and thyroid dysfunction. As a consequence, we failed to detect the involvement of any of these genes in thyroid dysfunction. Several explanations are possible: (A) the genes are not at all involved in thyroid dysfunction, (B) the SNP does not impact the action of sunitinib, and (C) the patient series is too small to detect such impact.

\section{Clinical impact of the correlation with rs2231142 in $A B C G 2$}

Our findings on the impact of rs2231142 in ABCG2 are interesting for two reasons. (A) It is interesting that our findings are in line with former publications because there have been difficulties in SNP research to validate findings in independent patient series [34]. (B) Secondly, our findings are important for a better understanding and prevention of interactions between distinct therapeutic molecules that are substrates of the efflux pump ABCG2. We have found additional evidence that sunitinib is a substrate of ABCG2. As a consequence, the intake of sunitinib could influence plasmatic levels of other medicines that are also effluxed by ABCG2 and vice versa.

\section{Conclusion}

Polymorphism rs2231142 in the efflux pump ABCG2 is associated with hypothyroidism observed in mRCC patients treated with sunitinib.

\section{Acknowledgments}

Pfizer supported this study with a research grant. Benoit Beuselinck received a grant from Fonds voor Wetenschappelijk Onderzoek Vlaanderen (Belgium). Diether Lambrechts is supported by the Stichting Tegen Kanker. He received research support from Hoffman-LaRoche and Sanofi-Aventis to conduct research projects related to the discovery of biomarkers for bevacizumab therapy. Evelyne Lerut received funding from Fonds voor Wetenschappelijk Onderzoek Vlaanderen (Belgium) and Stichting tegen Kanker. Annelies Verbiest received an Emmanuel vander Schueren grant from Stichting Kom op tegen Kanker.

\section{Disclosure statement}

Diether Lambrechts served on advisory boards from Roche, Sanofi, Bayer, Novartis, Boehringer, and Eli-Lilly and received honoraria for this. Benoit Beuselinck received honoraria from Amgen, Pfizer, Janssen, and Bayer. Benoit Beuselinck is an investigator of the EudraCT: 2011-006085-40/MetaSun trial supported by Pfizer. The other authors have no conflicts of interest to declare.

\section{ORCID}

Emilie Werbrouck (D) http://orcid.org/0000-0002-0552-4095 Julie Bastin (D) http://orcid.org/0000-0001-8266-3008

Vincent Verschaeve (D) http://orcid.org/0000-0002-0575-6304

\section{References}

[1] Motzer RJ, Hutson TE, Tomczak P, et al. Sunitinib versus interferon alfa in metastatic renal-cell carcinoma. $\mathrm{N}$ Engl J Med. 2007;356(2):115-124.

[2] Motzer RJ, Hutson TE, Tomczak P, et al. Overall survival and updated results for sunitinib compared with interferon alfa in patients with metastatic renal cell carcinoma. J Clin Oncol. 2009;27(22):3584-3590.

[3] Wolter P, Stefan C, Decallonne B, et al. The clinical implications of sunitinib-induced hypothyroidism: a prospective evaluation. Br J Cancer. 2008;99(3):448454. 
[4] Garfield DH, Wolter P, Schoffski P, et al. Documentation of thyroid function in clinical studies with sunitinib: why does it matter? J Clin Oncol. 2008;26(31):51315132; author reply 2-3.

[5] Motzer RJ, Hutson TE, Cella D, et al. Pazopanib versus sunitinib in metastatic renal-cell carcinoma. $\mathrm{N}$ Engl J Med. 2013;369(8):722-731.

[6] Rini BI, Escudier B, Tomczak P, et al. Comparative effectiveness of axitinib versus sorafenib in advanced renal cell carcinoma (AXIS): a randomised phase 3 trial. Lancet. 2011;378(9807):1931-1939.

[7] Choueiri TK, Escudier B, Powles T, et al. Cabozantinib versus everolimus in advanced renal cell carcinoma (METEOR): final results from a randomised, openlabel, phase 3 trial. Lancet Oncol. 2016;17(7):917-927.

[8] Rini BI, Tamaskar I, Shaheen P, et al. Hypothyroidism in patients with metastatic renal cell carcinoma treated with sunitinib. J Natl Cancer Inst. 2007;99(1):81-83.

[9] Rogiers A, Wolter P, Op de Beeck K, et al. Shrinkage of thyroid volume in sunitinib-treated patients with renal-cell carcinoma: a potential marker of irreversible thyroid dysfunction? Thyroid. 2010;20(3):317-322.

[10] Ahmadieh H, Salti I. Tyrosine kinase inhibitors induced thyroid dysfunction: a review of its incidence, pathophysiology, clinical relevance, and treatment. Biomed Res Int. 2013;2013:725410.

[11] Brozik A, Hegedus C, Erdei Z, et al. Tyrosine kinase inhibitors as modulators of ATP binding cassette multidrug transporters: substrates, chemosensitizers or inducers of acquired multidrug resistance? Expert Opin Drug Metab Toxicol. 2011;7(5):623-642.

[12] Haouala A, Widmer N, Duchosal MA, et al. Drug interactions with the tyrosine kinase inhibitors imatinib, dasatinib, and nilotinib. Blood. 2011;117(8):e75-e87.

[13] Shukla S, Robey RW, Bates SE, et al. Sunitinib (Sutent, SU11248), a small-molecule receptor tyrosine kinase inhibitor, blocks function of the ATP-binding cassette $(\mathrm{ABC})$ transporters P-glycoprotein $(\mathrm{ABCB} 1)$ and ABCG2. Drug Metab Dispos. 2009;37(2):359-365.

[14] $\mathrm{Hu} \mathrm{S}$, Chen Z, Franke R, et al. Interaction of the multikinase inhibitors sorafenib and sunitinib with solute carriers and ATP-binding cassette transporters. Clin Cancer Res. 2009;15(19):6062-6069.

[15] Beuselinck B, Karadimou A, Lambrechts D, et al. Singlenucleotide polymorphisms associated with outcome in metastatic renal cell carcinoma treated with sunitinib. Br J Cancer. 2013;108(4):887-900.

[16] Beuselinck B, Lambrechts D, Van Brussel T, et al. Efflux pump ABCB1 single nucleotide polymorphisms and dose reductions in patients with metastatic renal cell carcinoma treated with sunitinib. Acta Oncol. 2014:1-10.

[17] Garcia-Donas J, Esteban E, Leandro-Garcia LJ, et al. Single nucleotide polymorphism associations with response and toxic effects in patients with advanced renal-cell carcinoma treated with first-line sunitinib: a multicentre, observational, prospective study. Lancet Oncol. 2011;12(12):1143-1150.

[18] Mizuno T, Fukudo M, Fukuda T, et al. The effect of ABCG2 genotype on the population pharmacokinetics of sunitinib in patients with renal cell carcinoma. Ther Drug Monit. 2014;36(3):310-316.

[19] Narjoz C, Cessot A, Thomas-Schoemann A, et al. Role of thelean body mass and of pharmacogenetic variants on the pharmacokinetics and pharmacodynamics of sunitinib in cancer patients. Invest New Drugs. 2015;33(1):257-268.
[20] Low SK, Fukunaga K, Takahashi A, et al. Association study of a functional variant on ABCG2 gene with sunitinib-induced severe adverse drug reaction. PLoS One. 2016;11(2):e0148177.

[21] Kim HR, Park HS, Kwon WS, et al. Pharmacogenetic determinants associated with sunitinib-induced toxicity and ethnic difference in Korean metastatic renal cell carcinoma patients. Cancer Chemother Pharmacol. 2013;72(4):825-835.

[22] Diekstra MH, Swen JJ, Boven E, et al. CYP3A5 and $\mathrm{ABCB} 1$ polymorphisms as predictors for sunitinib outcome in metastatic renal cell carcinoma. Eur Urol. 2015;68:621-629.

[23] Diekstra MH, Klumpen HJ, Lolkema MP, et al. Association analysis of genetic polymorphisms in genes related to sunitinib pharmacokinetics, specifically clearance of sunitinib and SU12662. Clin Pharmacol Therap. 2014;96(1):81-89.

[24] Beuselinck B, Karadimou A, Lambrechts D, et al. VEGFR1 single nucleotide polymorphisms associated with outcome in patients with metastatic renal cell carcinoma treated with sunitinib - a multicentric retrospective analysis. Acta Oncol. 2014;53(1):103-112.

[25] Eechoute K, van der Veldt AA, Oosting S, et al. Polymorphisms in endothelial nitric oxide synthase (eNOS) and vascular endothelial growth factor (VEGF) predict sunitinib-induced hypertension. Clin Pharmacol Ther. 2012;92(4):503-510.

[26] Khan MS, Pandith AA, Iqbal M, et al. Possible impact of RET polymorphism and its haplotypic association modulates the susceptibility to thyroid cancer. J Cell Biochem. 2015;116(8):1712-1718.

[27] Reumers J, De Rijk P, Zhao H, et al. Optimized filtering reduces the error rate in detecting genomic variants by short-read sequencing. Nat Biotechnol. 2012;30(1):6168.

[28] Chu YH, Li H, Tan HS, et al. Association of ABCB1 and FLT3 polymorphisms with toxicities and survival in Asian patients receiving sunitinib for renal cell carcinoma. PLoS One. 2015;10(8):e0134102.

[29] Mizuno T, Fukudo M, Terada T, et al. Impact of genetic variation in breast cancer resistance protein (BCRP/ ABCG2) on sunitinib pharmacokinetics. Drug Metab Pharmacokinet. 2012;27(6):631-639.

[30] Mizuno T, Terada T, Kamba T, et al. ABCG2 421C>A polymorphism and high exposure of sunitinib in a patient with renal cell carcinoma. Ann Oncol. 2010;21(6):1382-1383.

[31] van der Veldt AA, Eechoute K, Gelderblom H, et al. Genetic polymorphisms associated with a prolonged progression-free survival in patients with metastatic renal cell cancer treated with sunitinib. Clin Cancer Res. 2011;17(3):620-629.

[32] Teo YL, Wee HL, Chue XP, et al. Effect of the CYP3A5 and $\mathrm{ABCB} 1$ genotype on exposure, clinical response and manifestation of toxicities from sunitinib in Asian patients. Pharmacogenomics J. 2016;16(1):47-53.

[33] Numakura K, Tsuchiya N, Kagaya H, et al. Clinical effects of single nucleotide polymorphisms on drug-related genes in Japanese metastatic renal cell carcinoma patients treated with sunitinib. Anticancer Drugs. 2017;28(1):97-103.

[34] Beuselinck B, Zucman-Rossi J. Kidney cancer: single nucleotide polymorphisms in mRCC-is their time up? Nat Rev Urol. 2015;12(8):424-426. 